\title{
Fault Diagnosis Observer Design for Discrete-Time Delayed Complex Interconnected Networks with Linear Coupling
}

\author{
Zhanshan Wang, ${ }^{1}$ Huanxin Guan, ${ }^{2}$ and Chengde Zheng $^{3}$ \\ ${ }^{1}$ School of Information Science and Engineering, Northeastern University, Liaoning, \\ Shenyang 110004, China \\ ${ }^{2}$ Department of Electrical Engineering, Shenyang Institute of Engineering, Shenyang 110136, China \\ ${ }^{3}$ Department of Mathematics, Dalian Jiaotong University, Dalian 116028, China
}

Correspondence should be addressed to Zhanshan Wang, zhanshan_wang@163.com

Received 31 August 2012; Accepted 17 October 2012

Academic Editor: Bin Jiang

Copyright (C) 2012 Zhanshan Wang et al. This is an open access article distributed under the Creative Commons Attribution License, which permits unrestricted use, distribution, and reproduction in any medium, provided the original work is properly cited.

Fault diagnosis for a class of discrete-time delayed complex interconnected networks with linear coupling in the case of actuator fault is studied. For the case of unavailability of network state, a state observer is first designed. Then a fault diagnosis observer is designed to detect the actuator fault on the basis of online adaptive approximator, which can approximate the unmodeled dynamics of the complex networks. Lastly, by choosing a suitable threshold, the actuator fault can be detected. A numerical simulation is used to show the effectiveness of the proposed method.

\section{Introduction}

Fault diagnosis for dynamic systems has received more and more attention in the last decades due to the increasing demand for higher performance, higher safety and reliability standards. The main task of the fault diagnosis can be roughly described as the early detection and diagnosis of faulty elements in a system, as well as the time of the detection. Many fault diagnosis approaches have been reported in the existing literature [1-3]. All these proposed fault diagnosis approaches have been applied to practical processes successfully [4-11], to name a few. Among these techniques, one important type is the observer-based approach, where fault diagnosis observers are used to generate residual signals to detect the fault.

Model-based schemes have emerged as prominent approaches to fault diagnosis of continuous and discrete-time systems $[1,2,10-15]$. This approach is built on a mathematical model of the process that must be monitored, so that a number of residuals can be 
computed by taking the difference between the estimated value of the system output variables and their measured value. The residuals are then compared to suitable thresholds by detection and isolation logics in order to provide fault decisions regarding the health of the system [16-18]. Model-based approaches are well suited to monitor the centralized and small systems, but suffer from scalability and robustness issues when distributed and large-scale systems are concerned, for which there have been significant research activities recently [19]. In [20], a distributed fault detection scheme was introduced for a class of continuous-time systems based on an overlapping decomposition technique, in which the subsystems were monitored by a network of interconnected local fault diagnosis mechanism.

Note that all above concerned systems are mainly focused on isolated node systems, which are the counterparts of the complex interconnected network systems [21]. Over the past few years, complex networks have been gaining increasing research attention because of their potential applications in many real-world systems from a variety of fields such as biology, social systems, linguistic networks, and technological systems [22-27]. In particular, rapidly growing research results have been reported in the literature that have focused on the structural properties between the coupled nodes in the complex networks such as stability and robustness. As one of the mostly investigated dynamical behaviors, synchronization in complex networks with or without time delays has drawn significant research interest in recent years [22, 23, 26, 28, 29]. It is well known that the faults may happen in the complex network systems, which is composed of the same or different node system. Therefore, the fault diagnosis problem for the complex interconnected networks plays an important role in the fault diagnosis theory.

In the recent papers [30-32], the authors first studied the fault diagnosis problems in the complex interconnected networks, and the adaptive control methods were adopted to realize the fault-tolerant synchronization in the case of network deterioration. The main object in [30-32] is to adjust the coupling strength using the adaptive method. Obviously, this kind of deteriorated networks can have robust synchronization stability in the case of uncertainties. In [33], a state feedback-based decentralized control scheme was proposed for a class of networked large-scale systems with output sensor failures. However, the designed controller required all the state combination of the whole networks, which made the method in [33] restrictive in practice. In contrast to the fault diagnosis problem in the conventional fault diagnosis theory $[16,17]$, there are many problems to be resolved in the complex networks, for example, how to detect the fault in the isolated node system such as actuator fault and sensor fault. Such kind of problems is not studied deeply at present.

Motivated by the above discussions, this paper will study the fault diagnosis problem for a class of discrete-time complex interconnected networks in the presence of actuator fault. Considering the unavailability of the system states, a state observer is first constructed to estimate the states via the linear matrix inequality (LMI) method. When the system states are available, a fault diagnosis observer is designed based on an adaptive technique, in which the uncertainty is approximated by an online approximator. The adopted method is a generalization of the isolated node system to the complex system, in which the difficulty lies in dealing with the coupling of the interconnected subsystems. By constructing a set of suitable observers via the spirit of distributed control concept, we have discussed a special class of actuator faults in the complex system. A numerical example is used to show the effectiveness of the obtained result. 


\section{Problem Description and Preliminaries}

In this paper we will discuss the following complex interconnected networks with $N$ identical nodes:

$$
\begin{gathered}
x_{i}(k+1)=A x_{i}(k)+g\left(x_{i}(k)\right)+g\left(x_{i}(k-\tau(k))\right)+B\left(x_{i}(k)\right) u(k) \\
+\sum_{j=1}^{N} G_{i j} \Gamma x_{j}(k)+\eta\left(k, x_{i}(k), u(k)\right) \\
y_{i}(k)=D x_{i}(k)
\end{gathered}
$$

where $N \geq 1$ is a positive integer, $x_{i}(k)=\left(x_{i 1}(k), \ldots, x_{i n}(k)\right)^{T}$, and $n$ is the dimensional number of node system state $x_{i}(k) . A$ is the linearly dominated matrix with appropriate dimension, and $g\left(x_{i}(k)\right)=\left(g_{1}\left(x_{i 1}(k)\right), \ldots, g_{n}\left(x_{i n}(k)\right)\right)^{T}$ is the known nonlinear function. $B\left(x_{i}(k)\right)$ is the control input matrix; $u(k)$ is the control input. Positive integer $\tau(k)>0$ is the discrete time-varying delay satisfying $\tau_{m} \leq \tau(k) \leq \tau_{M}$, where $\tau_{m}, \tau_{M}$ are known integers and $\Gamma$ is a positive diagonal matrix. $G=\left(G_{i j}\right) \in \mathbb{R}^{N \times N}$ is the coupled configuration matrix of the networks with $G_{i j}>0(i \neq j)$ but not all zeros, $\sum_{l=1}^{N} G_{s l}=\sum_{l=1}^{N} G_{l s}=0, s=1, \ldots, N$, $G=G^{T}, \eta\left(k, x_{i}(k), u(k)\right)$ denotes the disturbances and uncertainties, $i=1, \ldots, N . y_{i}(k)$ is the system output, and $D$ is the system output matrix with appropriate dimension.

Assumption 2.1. The nonlinear function $g_{i}(\eta)$ is bounded and continuous, which satisfies $\left|g_{i}(\eta)\right| \leq G_{i}^{b}$, where $G_{i}^{b}>0$ is a positive constant,

$$
\left[g(\eta)-g(v)-\Delta_{1}(\eta-v)\right]^{T}\left[g(\eta)-g(v)-\Delta_{2}(\eta-v)\right] \leq 0,
$$

for any $\eta \neq v, \eta, v \in \mathfrak{R}^{n}$, and $\Delta_{1}$ and $\Delta_{2}$ are constant matrices.

Note that condition (2.3) can be changed into the following form:

$$
\left[(\eta-v)^{T}(g(\eta)-g(v))^{T}\right]\left[\begin{array}{cc}
\Delta_{1}^{T} \Delta_{2} & -\Delta_{1}^{T} \\
-\Delta_{2} & I
\end{array}\right]\left[\begin{array}{c}
\eta-v \\
g(\eta)-g(v)
\end{array}\right] \leq 0
$$

or

$$
\left[(\eta-v)^{T}(g(\eta)-g(v))^{T}\right]\left[\begin{array}{cc}
\Delta_{2}^{T} \Delta_{1} & -\Delta_{2}^{T} \\
-\Delta_{1} & I
\end{array}\right]\left[\begin{array}{c}
\eta-v \\
g(\eta)-g(v)
\end{array}\right] \leq 0
$$

Combining inequalities (2.4) and (2.5), we have

$$
\left[(\eta-v)^{T}(g(\eta)-g(v))^{T}\right]\left[\begin{array}{cc}
\Delta_{2}^{T} \Delta_{1}+\Delta_{1}^{T} \Delta_{2} & -\Delta_{2}^{T}-\Delta_{1}^{T} \\
-\Delta_{1}-\Delta_{2} & 2 I
\end{array}\right]\left[\begin{array}{c}
\eta-v \\
g(\eta)-g(v)
\end{array}\right] \leq 0 .
$$

It should be noticed that the above model (2.1)-(2.2) may represent the discrete-time equivalent of a continuous model obtained via the well-known Euler method. The class of failure considered in this work is that of actuator fault. This class of failure can be represented 
as an unknown additive disturbance on the nominal input to the system $\bar{u}(k)$. Hence, an actuator fault occurring at the $k$ th time step results in a faulty input given by

$$
u(k)=\bar{u}(k)+\delta u(k)
$$

where $\delta u(k)$ represents the time profile of the unknown fault. Therefore, the nominal dynamics (2.1) in the presence of faults becomes

$$
\begin{aligned}
x_{i}(k+1)= & A x_{i}(k)+g\left(x_{i}(k)\right)+g\left(x_{i}(k-\tau(k))\right)+B\left(x_{i}(k)\right) \bar{u}(k) \\
& +\sum_{j=1}^{N} G_{i j} \Gamma x_{j}(k)+\eta\left(k, x_{i}(k), \bar{u}(k), \theta(k)\right)+f\left(k, x_{i}(k)\right),
\end{aligned}
$$

where the fault vector $f\left(k, x_{i}(k)\right)$ is given by $f\left(k, x_{i}(k)\right)=B\left(x_{i}(k)\right) \delta u(k)$.

The uncertain term $\eta\left(k, x_{i}(k), \bar{u}(k), \theta(k)\right)$ is assumed to depend on the nominal input and on the parameter vector $\theta(k)$. If $\eta\left(k, x_{i}(k), \bar{u}(k), \theta(k)\right)$ is linear in the parameter vector, it can be expressed as $\eta\left(k, x_{i}(k), \bar{u}(k), \theta(k)\right)=\Omega\left(k, x_{i}(k), \bar{u}(k)\right) \theta(k)$, where the matrix $\Omega(\cdot)$ is assumed to be known, while $\theta(k)$ is usually unknown (or partially known). If $\theta(k)$ is not linear in the parameter or its structure is not exactly known, an approximation scheme can be obtained by resorting to the so-called online interpolators [34-38] (e.g., neural networks, fuzzy logic, and splines). By choosing a linear-in-the-parameters interpolator structure, the uncertain term can be expressed as follows:

$$
\eta\left(k, x_{i}(k), \bar{u}(k), \theta(k)\right)=\Omega\left(k, x_{i}(k), \bar{u}(k)\right) \theta(k)+\varepsilon\left(k, x_{i}(k), \bar{u}(k)\right),
$$

where $\varepsilon\left(k, x_{i}(k), \bar{u}(k)\right)$ represents the interpolation error.

The following assumption is required.

Assumption 2.2. $B\left(x_{i}(k)\right)$ is a column full rank matrix, $i=1, \ldots, N$.

Assumption 2.3. The norm of the matrix $\Omega\left(k, x_{i}(k), \bar{u}(k)\right)$ is uniformly bounded by a constant $\Omega>0$, and the norm of the interpolation error $\varepsilon\left(k, x_{i}(k), \bar{u}(k)\right)$ is uniformly bounded by a constant $\varepsilon_{0}>0$.

Usually, the network output is a linear combination of the information about the network nodes. We wish to design an observer to estimate the network states through the available network output. In order to estimate the states, the following state observer is designed, $i=1, \ldots, N$ :

$$
\begin{aligned}
\widehat{x}_{i}(k+1)= & A \widehat{x}_{i}(k)+g\left(\widehat{x}_{i}(k)\right)+g\left(\widehat{x}_{i}(k-\tau(k))\right)+B\left(\widehat{x}_{i}(k)\right) \bar{u}(k) \\
& +\sum_{j=1}^{N} G_{i j} \Gamma \widehat{x}_{j}(k)+\widehat{\eta}\left(k, \widehat{x}_{i}(k), \bar{u}(k), \hat{\theta}\right)-K\left(y_{i}(k)-D \widehat{x}_{i}(k)\right) .
\end{aligned}
$$


Therefore, in view of (2.8) and (2.10), the state estimation error is given by

$$
\begin{aligned}
e_{i}(k+1)= & (A+K D) e_{i}(k)+\tilde{g}\left(e_{i}(k)\right)+\tilde{g}\left(e_{i}(k-\tau(k))\right)+\widetilde{B}\left(e_{i}(k)\right) \bar{u}(k) \\
& +\sum_{j=1}^{N} G_{i j} \Gamma e_{j}(k)+\tilde{\eta}\left(k, \widehat{x}_{i}(k), \bar{u}(k), \widehat{\theta}\right)+f\left(k, \widehat{x}_{i}(k)\right),
\end{aligned}
$$

where $e_{i}(k)=x_{i}(k)-\widehat{x}_{i}(k), \tilde{g}\left(e_{i}(k-\tau(k))\right)=g\left(x_{i}(k-\tau(k))\right)-g\left(\widehat{x}_{i}(k-\tau(k))\right), \widetilde{B}\left(e_{i}(k)\right)=$ $B\left(x_{i}(k)\right)-B\left(\widehat{x}_{i}(k)\right), \tilde{g}\left(e_{i}(k)\right)=g\left(x_{i}(k)\right)-g\left(\widehat{x}_{i}(k)\right), \widetilde{\eta}\left(k, \widehat{x}_{i}(k), \bar{u}(k), \hat{\theta}\right)=\eta\left(k, x_{i}(k), \bar{u}(k), \widehat{\theta}\right)-$ $\widehat{\eta}\left(k, \widehat{x}_{i}(k), \bar{u}(k), \widehat{\theta}\right), i=1, \ldots, N$.

Before we give the main results, we need the following preliminaries.

Lemma 2.4 (see $[26,28]$ ). Let $U=\left(u_{i j}\right)_{N \times N}, P \in R^{n \times n}, \alpha=\left(\alpha_{1}^{T}, \alpha_{2}^{T}, \ldots, \alpha_{N}^{T}\right)^{T}, \gamma=$ $\left(\gamma_{1}^{T}, \gamma_{2}^{T}, \ldots, \gamma_{N}^{T}\right)^{T}, \alpha_{k} \in R^{n \times n}, \gamma_{k} \in R^{n \times n}, k=1, \ldots, N$. If $\mathcal{U}=\mathfrak{U}^{T}$ and each row sum of $\mathcal{U}$ is zero, then

$$
\alpha^{T}(\mathcal{U} \otimes P) \gamma=-\sum_{1 \leq i<j \leq N} u_{i j}\left(\alpha_{i}-\alpha_{j}\right)^{T} P\left(\gamma_{i}-\gamma_{j}\right)
$$

where symbol $\otimes$ denotes the Kronecker product.

Lemma 2.5 (Schur complement, see $[22,27]$ ). Given constant matrices $S_{1}, S_{2}$, and $S_{3}$, where $S_{1}=S_{1}^{T}$ and $S_{2}>0$ is a positive definite symmetric matrix, then

$$
S_{1}+S_{3}^{T} S_{2}^{-1} S_{3}<0
$$

if and only if

$$
\left[\begin{array}{cc}
S_{1} & S_{3}^{T} \\
S_{3} & -S_{2}
\end{array}\right]<0
$$

Lemma 2.6 (see $[22,29]$ ). Let $b \in \mathbb{R}$ and $A, B, C, D$ be matrices with appropriate dimensions. The following statements about Kronecker product are true:

(1) $b(A \otimes B)=(b A) \otimes B=A \otimes(b B)$;

(2) $(A \otimes B)^{T}=A^{T} \otimes B^{T}$;

(3) $(A \otimes B)(C \otimes D)=(A C) \otimes(B D)$;

(4) $A \otimes B \otimes C=(A \otimes B) \otimes C=A \otimes(B \otimes C)$;

(5) $(A+B) \otimes(C+D)=A \otimes C+B \otimes C+A \otimes D+B \otimes D$.

\section{State Observer Design}

Now we state our main results in this section.

Suppose that the online approximator has met the precision of modelling of the uncertainty, then $\tilde{\eta}(k)$ approaches to zero. For the case of no fault and no approximation 
error in (2.11), that is, $\varepsilon(k)=0, f\left(k, \widehat{x}_{i}(k)\right)=0$, we will study the stability of the following state observer, $i=1, \ldots, N$ :

$$
e_{i}(k+1)=\bar{A} e_{i}(k)+\tilde{g}\left(e_{i}(k)\right)+\tilde{g}\left(e_{i}(k-\tau(k))\right)+\sum_{j=1}^{N} G_{i j} \Gamma e_{j}(k),
$$

or in a compact form

$$
e(k+1)=(I \otimes \bar{A}) e(k)+g_{c}(e(k))+g_{c}(e(k-\tau(k)))+(G \otimes \Gamma) e(k),
$$

where $e(k)=\left(e_{1}^{T}(k), e_{2}^{T}(k), \ldots, e_{N}^{T}(k)\right)^{T}, g_{c}(e(k))=\left(\tilde{g}^{T}\left(e_{1}(k)\right), \ldots, \tilde{g}^{T}\left(e_{N}(k)\right)\right)^{T}, \bar{A}=(A+$ $K D)$.

Theorem 3.1. Suppose that Assumption 2.1 holds. The observer error system (3.1) or (3.2) is globally asymptotically stable if, for a given observer gain matrix $K$, there exist positive constant $\alpha$, symmetric positive definite matrices $P$ and $Q$ with appropriate dimensions, such that the following condition holds, $1 \leq i<j \leq N$ :

$$
\Phi_{i j}=\left[\begin{array}{ccc}
\Phi_{i j}^{1} & \Phi_{i j}^{2} & \bar{A}^{T} P^{T}-N G_{i j} \Gamma P^{T} \\
* & P+\left(1+\tau_{M}-\tau_{m}\right) Q-2 \alpha I & P \\
* & * & P-Q
\end{array}\right]<0
$$

where I is an identity matrix with appropriate dimension,

$$
\begin{gathered}
\Phi_{i j}^{1}=\bar{A}^{T} P \bar{A}-P-N G_{i j} \bar{A}^{T} P \Gamma-\left(N G_{i j} \bar{A}^{T} P \Gamma\right)^{T}-N G_{i j}^{(2)} \Gamma P \Gamma-\alpha\left(\Delta_{2}^{T} \Delta_{1}+\Delta_{1}^{T} \Delta_{2}\right), \\
\Phi_{i j}^{2}=\bar{A}^{T} P-N G_{i j} \Gamma P^{T}+\alpha\left(\Delta_{1}+\Delta_{2}\right) . \\
G_{i j}^{(2)} \text { is the }(i, j) \text { elements of matrix } G^{T} G=G^{2} .
\end{gathered}
$$

Proof. Let us consider the Lyapunov functional $V(k)=V_{1}(k)+V_{2}(k)+V_{3}(k)$, where

$$
\begin{gathered}
V_{1}(k)=e^{T}(k)(U \otimes P) e(k), \\
V_{2}(k)=\sum_{i=k-\tau(k)}^{k-1} g_{c}^{T}(e(i))(U \otimes Q) g_{c}(e(i)), \\
V_{3}(k)=\sum_{j=k-\tau_{M}+1}^{k-\tau_{m}} \sum_{i=j}^{k-1} g_{c}^{T}(e(i))(U \otimes Q) g_{c}(e(i)),
\end{gathered}
$$


where

$$
U=\left[\begin{array}{cccc}
N-1 & -1 & \cdots & -1 \\
-1 & N-1 & \cdots & -1 \\
\cdots & \cdots & \cdots & \cdots \\
-1 & -1 & \cdots & N-1
\end{array}\right]_{N \times N}
$$

Calculating the difference of $V(k)$ along the system (3.2), we have

$$
\Delta V(k)=\Delta V_{1}(k)+\Delta V_{2}(k)+\Delta V_{3}(k)
$$

where

$$
\begin{aligned}
\Delta V_{1}(k)= & V_{1}(k+1)-V_{1}(k) \\
= & \left((I \otimes \bar{A}) e(k)+g_{c}(e(k))+g_{c}(e(k-\tau(k)))+(G \otimes \Gamma) e(k)\right)^{T} \\
& \times(U \otimes P)\left((I \otimes \bar{A}) e(k)+g_{c}(e(k))+g_{c}(e(k-\tau(k)))+(G \otimes \Gamma) e(k)\right) \\
& -e^{T}(k)(U \otimes P) e(k), \\
= & e^{T}(k)(I \otimes \bar{A})^{T}(U \otimes P)(I \otimes \bar{A}) e(k)+g_{c}^{T}(e(k))(U \otimes P) g_{c}(e(k)) \\
& +g_{c}^{T}(e(k-\tau(k)))(U \otimes P) g_{c}(e(k-\tau(k)))-e^{T}(k)(U \otimes P) e(k) \\
& +e^{T}(k)(G \otimes \Gamma)^{T}(U \otimes P)(G \otimes \Gamma) e(k) \\
& +2 g_{c}^{T}(e(k))(U \otimes P)(I \otimes \bar{A}) e(k)+2 g_{c}^{T}(e(k))(U \otimes P) g_{c}(e(k-\tau(k))) \\
& +2 g_{c}^{T}(e(k))(U \otimes P)(G \otimes \Gamma) e(k)+2 g_{c}^{T}(e(k-\tau(k)))(U \otimes P)(I \otimes \bar{A}) e(k) \\
& +2 g_{c}^{T}(e(k-\tau(k)))(U \otimes P)(G \otimes \Gamma) e(k) \\
& +2 e^{T}(k)(I \otimes \bar{A})^{T}(U \otimes P)(G \otimes \Gamma) e(k), \\
& -\sum_{i=k+1-\tau(k)}^{k-1} g_{c}^{T}(e(i))(U \otimes Q) g_{c}(e(i))-g_{c}^{T}(e(k-\tau(k)))(U \otimes Q) g_{c}(e(k-\tau(k))), \\
\Delta V_{2}(k)= & V_{2}(k+1)-V_{2}(k), \\
& \sum_{i=}^{k} g_{i-1-\tau(k+1)} g_{c}^{T}(e(i))(U \otimes Q) g_{c}(e(i))-\sum_{i=k-\tau(k)}^{k-1} g_{c}^{T}(e(i))(U \otimes Q) g_{c}(e(i)), \\
= & g_{c}^{T}(e(k))(U \otimes Q) g_{c}^{T}(e(i))(U \otimes Q) g_{c}(e(i)) \\
& (k)+1-\tau(k+1)
\end{aligned}
$$




$$
\begin{aligned}
= & g_{c}^{T}(e(k))(U \otimes Q) g_{c}(e(k))+\sum_{i=k+1-\tau(k+1)}^{k-\tau_{m}} g_{c}^{T}(e(i))(U \otimes Q) g_{c}(e(i)) \\
& +\sum_{i=k-\tau_{m}+1}^{k-1} g_{c}^{T}(e(i))(U \otimes Q) g_{c}(e(i)) \\
& -\sum_{i=k+1-\tau(k)}^{k-1} g_{c}^{T}(e(i))(U \otimes Q) g_{c}(e(i))-g_{c}^{T}(e(k-\tau(k)))(U \otimes Q) g_{c}(e(k-\tau(k))), \\
\leq & g_{c}^{T}(e(k))(U \otimes Q) g_{c}(e(k))-g_{c}^{T}(e(k-\tau(k)))(U \otimes Q) g_{c}(e(k-\tau(k))) \\
& +\sum_{i=k+1-\tau_{M}}^{k-\tau_{m}} g_{c}^{T}(e(i))(U \otimes Q) g_{c}(e(i)), \\
= & V_{3}(k+1)-V_{3}(k), \\
= & \sum_{j=k-\tau_{M}+2}^{k-\tau_{m}+1} \sum_{i=j}^{k} g_{c}^{T}(e(i))(U \otimes Q) g_{c}(e(i))-\sum_{j=k-\tau_{M}+1}^{k-\tau_{m}} \sum_{i=j}^{k-1} g_{c}^{T}(e(i))(U \otimes Q) g_{c}(e(i)), \\
= & \sum_{j=k-\tau_{M}+1 i=j+1}^{k-\tau_{m}} \sum_{j}^{k} g_{c}^{T}(e(i))(U \otimes Q) g_{c}(e(i))-\sum_{j=k-\tau_{M}+1}^{k-\tau_{m}} \sum_{i=j}^{k-1} g_{c}^{T}(e(i))(U \otimes Q) g_{c}(e(i)), \\
= & \sum_{j=k-\tau_{M}+1}^{k-\tau_{m}}\left(g_{c}^{T}(e(k))(U \otimes Q) g_{c}(e(k))-g_{c}^{T}(e(j))(U \otimes Q) g_{c}(e(j))\right), \\
\leq & \left.\tau_{M}-\tau_{m}\right) g_{c}^{T}(e(k))(U \otimes Q) g_{c}(e(k))-\sum_{i=k-\tau_{M}+1}^{k-\tau_{m}} g_{c}^{T}(e(i))(U \otimes Q) g_{c}(e(i)) .
\end{aligned}
$$

Note that the following equalities hold according to Lemma 2.6:

$$
\begin{aligned}
(G \otimes \Gamma)^{T}(U \otimes P)(G \otimes \Gamma) & =\left(G^{T} \otimes \Gamma^{T}\right)(U \otimes P)(G \otimes \Gamma) \\
& =\left(G^{T} U G\right) \otimes\left(\Gamma^{T} P \Gamma\right) \\
& =N G^{2} \otimes(\Gamma P \Gamma), \\
(I \otimes \bar{A})^{T}(U \otimes P)(I \otimes \bar{A}) & =\left(I \otimes \bar{A}^{T}\right)(U \otimes P)(I \otimes \bar{A}) \\
& =U \otimes\left(\bar{A}^{T} P \bar{A}\right), \\
(I \otimes \bar{A})^{T}(U \otimes P)(G \otimes \Gamma) & =N G \otimes\left(\bar{A}^{T} P \Gamma\right), \\
(U \otimes P)(G \otimes \Gamma) & =N G \otimes(P \Gamma) .
\end{aligned}
$$


Mathematical Problems in Engineering

Substituting (3.8) and (3.9) into (3.7), it yields

$$
\begin{aligned}
\Delta V(k) \leq & e^{T}(k)\left(U \otimes\left(\bar{A}^{T} P \bar{A}\right)\right) e(k)+g_{c}^{T}(e(k))(U \otimes P) g_{c}(e(k)) \\
& +g_{c}^{T}(e(k-\tau(k)))(U \otimes P) g_{c}(e(k-\tau(k)))-e^{T}(k)(U \otimes P) e(k) \\
& +e^{T}(k)\left(N G^{2} \otimes(\Gamma P \Gamma)\right) e(k) \\
& +2 g_{c}^{T}(e(k))(U \otimes(P \bar{A})) e(k)+2 g_{c}^{T}(e(k))(U \otimes P) g_{c}(e(k-\tau(k))) \\
& +2 g_{c}^{T}(e(k))(N G \otimes(P \Gamma)) e(k)+2 g_{c}^{T}(e(k-\tau(k)))(U \otimes(P \bar{A})) e(k) \\
& +2 g_{c}^{T}(e(k-\tau(k)))(N G \otimes(P \Gamma)) e(k) \\
& +2 e^{T}(k)\left(N G \otimes\left(\bar{A}^{T} P \Gamma\right)\right) e(k) \\
& +\left(1+\tau_{M}-\tau_{m}\right) g_{c}^{T}(e(k))(U \otimes Q) g_{c}(e(k)) \\
& -g_{c}^{T}(e(k-\tau(k)))(U \otimes Q) g_{c}(e(k-\tau(k))) .
\end{aligned}
$$

According to Lemma 2.4 and inequality (2.6), (3.10) can be changed into the following form:

$$
\begin{aligned}
\Delta V(k) \leq \sum_{1 \leq i<j \leq N}( & e_{i j}^{T}(k) \bar{A}^{T} P \bar{A} e_{i j}(k)+\widetilde{g}_{i j}^{T}(e(k)) P \widetilde{g}_{i j}(e(k)) \\
& +\tilde{g}_{i j}^{T}(e(k-\tau(k))) P \widetilde{g}_{i j}(e(k-\tau(k)))-e_{i j}^{T}(k) P e_{i j}(k) \\
& -e_{i j}^{T}(k)\left(N G_{i j}^{(2)} \Gamma P \Gamma\right) e_{i j}(k) \\
& +2 \widetilde{g}_{i j}^{T}(e(k)) P \bar{A} e_{i j}(k)+2 \tilde{g}_{i j}^{T}(e(k)) P \widetilde{g}_{i j}(e(k-\tau(k))) \\
& -2 \tilde{g}_{i j}^{T}(e(k)) N G_{i j} P \Gamma e_{i j}(k)+2 \tilde{g}_{i j}^{T}(e(k-\tau(k))) P \bar{A} e_{i j}(k) \\
& -2 \widetilde{g}_{i j}^{T}(e(k-\tau(k))) N G_{i j} P \Gamma e_{i j}(k) \\
& -2 e^{T}(k) N G_{i j} \bar{A}^{T} P \Gamma e_{i j}(k) \\
& +\left(1+\tau_{M}-\tau_{m}\right) \tilde{g}_{i j}^{T}(e(k)) Q \tilde{g}_{i j}(e(k))
\end{aligned}
$$




$$
\begin{gathered}
-\tilde{g}_{i j}^{T}(e(k-\tau(k))) Q \tilde{g}_{i j}(e(k-\tau(k))) \\
\left.-\alpha\left[e_{i j}^{T}(k) \tilde{g}_{i j}^{T}(e(k))\right]\left[\begin{array}{cc}
\Delta_{2}^{T} \Delta_{1}+\Delta_{1}^{T} \Delta_{2} & -\Delta_{2}^{T}-\Delta_{1}^{T} \\
-\Delta_{1}-\Delta_{2} & 2 I
\end{array}\right]\left[\begin{array}{c}
e_{i j}(k) \\
\tilde{g}_{i j}(e(k))
\end{array}\right]\right), \\
=\sum_{1 \leq i<j \leq N} \xi_{i j}^{T}(k) \Phi_{i j} \xi_{i j}(k), \\
\leq \sum_{1 \leq i<j \leq N} \lambda_{\max }\left(\Phi_{i j}\right)\left\|\xi_{i j}(k)\right\|^{2},
\end{gathered}
$$

where $\alpha>0$ is a free parameter to be adjusted, $G^{2}=G^{T} G=\left(G_{i j}^{(2)}\right)_{N \times N}, \lambda_{\max }\left(\Phi_{i j}\right)$ denotes the maximum eigenvalue of a matrix $\Phi_{i j}, e_{i j}(k)=\left(e_{i}(k)-e_{j}(k)\right), \tilde{g}_{i j}(e(k))=g_{c i}(e(k))-g_{c j}(e(k))$, $\tilde{g}_{i j}(e(k-\tau(k)))=g_{c i}(e(k-\tau(k)))-g_{c j}(e(k-\tau(k)))$, and $\xi_{i j}(k)=\left(e_{i j}^{T}(k), \tilde{g}_{i j}^{T}(e(k)), \tilde{g}_{i j}^{T}(e(k-\right.$ $\tau(k))))^{T}$. Considering the negativeness of matrix $\Phi_{i j}$ in (3.3), we have $\lambda_{\max }\left(\Phi_{i j}\right)<0$. Letting $\lambda_{0}=\max _{1 \leq i<j \leq N}\left\{\lambda_{\max }\left(\Phi_{i j}\right)\right\}$, we have $\lambda_{0}<0$, and then

$$
\Delta V(k) \leq \lambda_{0} \sum_{1 \leq i<j \leq N}\left\|e_{i j}(k)\right\|^{2}<0 .
$$

Let $m$ be a positive integer; then from (3.12) one has

$$
V(m+1)-V(1)=\sum_{k=1}^{m} \Delta V(k)=\lambda_{0} \sum_{1 \leq i<j \leq N k=1} \sum^{m}\left\|e_{i j}(k)\right\|^{2},
$$

which implies that

$$
-\lambda_{0} \sum_{1 \leq i<j \leq N} \sum_{k=1}^{m}\left\|e_{i j}(k)\right\|^{2} \leq V(1)
$$

By letting $m \rightarrow+\infty$, we can deduce that the series $\sum_{k=1}^{+\infty}\left\|e_{i j}(k)\right\|^{2}$ is convergent for $1 \leq i<j \leq$ $N$, and therefore, $\left\|e_{i j}(k)\right\|^{2} \rightarrow 0$, namely, $\operatorname{limit}_{k \rightarrow+\infty}\left|x_{i}(k)-\widehat{x}_{i}(k)\right|=0$, which completes the proof of Theorem 3.1.

In order to solve the observer gain matrix $K$, according to Lemma 2.5, we have the following result.

Theorem 3.2. Suppose that Assumption 2.1 holds. The observer error system (3.1) or (3.2) is globally asymptotically stable if there exist positive constant $\alpha$, symmetric positive definite matrices $P$ and $Q$ with appropriate dimensions, and appropriately dimensioned matrix $Y$, such that the following condition holds, $1 \leq i<j \leq N$ :

$$
\bar{\Phi}_{i j}=\left[\begin{array}{cccc}
\bar{\Phi}_{i j}^{1} & \bar{\Phi}_{i j}^{2} & \Phi_{i j}^{3} & A^{T} P+D^{T} Y^{T} \\
* & P+\left(1+\tau_{M}-\tau_{m}\right) Q-2 \alpha I & P & 0 \\
* & * & P-Q & 0 \\
* & * & * & -P
\end{array}\right]<0,
$$


where

$$
\begin{gathered}
\Phi_{i j}^{1}=-P-N G_{i j}\left(A^{T} P+D^{T} Y^{T}\right) \Gamma-N G_{i j} \Gamma(P A+Y D)-N G_{i j}^{(2)} \Gamma P \Gamma-\alpha\left(\Delta_{2}^{T} \Delta_{1}+\Delta_{1}^{T} \Delta_{2}\right), \\
\Phi_{i j}^{2}=A^{T} P+D^{T} Y^{T}-N G_{i j} \Gamma P^{T}+\alpha\left(\Delta_{1}+\Delta_{2}\right), \\
\Phi_{i j}^{3}=A^{T} P+D^{T} Y^{T}-N G_{i j} \Gamma P
\end{gathered}
$$

and the observer gain matrix $K=P^{-1} Y$.

Remark 3.3. Note that we are mainly concerned with the complex interconnected networks with identical node system in this paper. This kind of complex system can represent many practical systems such as secure communication, chaos generators design, and harmonic oscillation generation [23-26] (and references cited therein). Synchronization problems for such kind of complex networks have been paid much attention in recent years. However, for the fault diagnosis problem, there are few results to be reported. As a complex dynamical system, it is inevitable for a fault to occur in the normal operation. Therefore, combining the knowledge of fault diagnosis theory for isolated node system and the complex networks theory, the authors try to extend the diagnosis method in node system to the complex system. Correspondingly, we only consider a special kind of faults, that is, actuator fault, which has the same form in each node system. The key point of the proposed method lies in the choice of suitable state observer and diagnosis observer. For other kinds of faults, such as sensor faults, parameter faults, and hybrid faults, it still needs to be further studied in the future.

Remark 3.4. The LMI condition in Theorem 3.1 requires the information of the interconnected couplings $G_{i j}, \Gamma$, the node system parameters $A, D$ besides a given common observer gain matrix $K$. For a prescribed matrix $K$, if the LMI condition (3.3) holds, the designed observer exists and an estimated state can be achieved. In contrast, Theorem 3.2 aims to present an observer gain matrix $K$ directly, which has the same effect on the state estimation.

\section{Adaptive Observer-Based Fault Diagnosis}

Since all the states of the concerned system can be measured, in this section, we will consider the following fault diagnosis observer, which is used to detect the fault, $i=1, \ldots, N$ :

$$
\begin{aligned}
\widehat{x}_{f i}(k+1)= & A \widehat{x}_{f i}(k)+g\left(x_{i}(k)\right)+g\left(x_{i}(k-\tau(k))\right)+B\left(x_{i}(k)\right) \bar{u}(k) \\
& +\sum_{j=1}^{N} G_{i j} \Gamma x_{j}(k)+\hat{\eta}\left(k, x_{i}(k), \bar{u}(k), \widehat{\theta}(k)\right)+K_{o}\left(x_{i}(k)-\widehat{x}_{f i}(k)\right) .
\end{aligned}
$$

In view of (2.8) and (4.1), the diagnosis error dynamics is given by

$$
e_{f i}(k+1)=\left(A-K_{o}\right) e_{f i}(k)+\widehat{\eta}\left(k, x_{i}(k), \bar{u}(k), \widehat{\theta}(k)\right)+f\left(k, x_{i}(k)\right)
$$


where $\tilde{\eta}\left(k, x_{i}(k), \bar{u}(k), \hat{\theta}(k)\right)=\eta\left(k, x_{i}(k), \bar{u}(k), \theta(k)\right)-\widehat{\eta}\left(k, x_{i}(k), \bar{u}(k), \hat{\theta}(k)\right)$ represents the uncertainties estimation error, $e_{f i}(k)=x_{i}(k)-\widehat{x}_{f i}(k)$, and $K_{o}$ is chosen to make the eigenvalues of $A-K_{o}$ in the unit circle, $i=1, \ldots, N$.

The residuals vector can be chosen as

$$
r_{i}(k+1)=e_{f i}(k+1)-\Lambda e_{f i}(k),
$$

which can be rewritten as

$$
r_{i}(k+1)=\tilde{\eta}\left(k, x_{i}(k), \bar{u}(k), \hat{\theta}(k)\right)+f\left(k, x_{i}(k)\right),
$$

where $\Lambda=A-K_{o}$.

Note that the residual vector is affected by the fault vector and the estimation error of the uncertain term. If an accurate estimation of uncertain term is achieved, the fault signature on the residual (i.e., its effect on the residuals) becomes more evident. If a parametric model of the uncertainties is available, an adaptive estimation algorithm of the unknown parameters can be set up. It is worth remarking that such a paradigm has been keenly exploited for adaptive fault identification. However, in this work the same concept is exploited in order to adaptively compensate for the uncertainties, so as to obtain small values of the residuals in the absence of faults. In this case, the uncertain term can be indirectly evaluated through the estimation of $\theta(k)$. Thus, an adaptive update law for the parameters estimate $\theta(k)$ can be chosen as

$$
\begin{gathered}
\hat{\theta}(k+1)=\hat{\theta}(k)+\Omega^{T}(k) \Lambda_{\theta}(k) r(k+1), \\
\Lambda_{\theta}(k)=2\left[\Omega(\mathrm{k}) \Omega^{T}(k)+\Omega_{\theta}\right]^{-1},
\end{gathered}
$$

where $\Omega_{\theta}$ is a positive definite symmetric matrix.

In view of (2.9), the uncertainties estimation error $\tilde{\eta}\left(k, \widehat{x}_{i}(k), \bar{u}(k), \hat{\theta}(k)\right)$ can be written as

$$
\tilde{\eta}(k, \widehat{\theta}(k))=\Omega(k) \widetilde{\theta}(k)+\varepsilon(k),
$$

where $\tilde{\theta}(k)=\theta(k)-\hat{\theta}(k)$ is the parameters estimation error.

Therefore, we have the following diagnosis error system:

$$
\begin{gathered}
e_{f i}(k+1)=\Lambda e_{f i}(k)+\Omega(k) \tilde{\theta}(k)+\varepsilon(k)+f\left(k, \widehat{x}_{i}(k)\right), \\
\tilde{\theta}(k+1)=\left(I-\Omega^{T}(k) \Lambda_{\theta} \Omega(k)\right) \tilde{\theta}(k)-\Omega^{T}(k) \Lambda_{\theta}(f(k)+\varepsilon(k)) .
\end{gathered}
$$


In order to implement the fault detection, we first consider the stability problem of diagnosis error system (4.8) in the absence of fault and interpolation error, that is,

$$
\begin{gathered}
e_{f i}(k+1)=\Lambda e_{f i}(k)+\Omega(k) \tilde{\theta}(k), \\
\tilde{\theta}(k+1)=\left(I-\Omega^{T}(k) \Lambda_{\theta} \Omega(k)\right) \tilde{\theta}(k), \quad i=1, \ldots, N .
\end{gathered}
$$

Theorem 4.1. Under Assumption 2.3, the diagnosis error system (4.9) is globally uniformly stable, and the error $e_{f i}(k)$ converges asymptotically to zero, $i=1, \ldots, N$.

Proof. Consider the following functional:

$$
V_{i}(k)=e_{f i}^{T}(k) S e_{f i}(k)+q \tilde{\theta}^{T}(k) \tilde{\theta}(k),
$$

where $q$ is a positive constant and $S$ is the solution to the Ricatti equation $S-\Lambda^{T} S \Lambda=Q_{0}$ for a given symmetric and positive definite matrix $Q_{0}$.

The difference of $V_{i}(k)$ can be calculated as follows, with the consideration of (4.6):

$$
\begin{aligned}
\Delta V_{i}(k)= & V_{i}(k+1)-V_{i}(k) \\
= & \left(\Lambda e_{f i}(k)+\Omega(k) \tilde{\theta}(k)\right)^{T}\left(\Lambda e_{f i}(k)+\Omega(k) \tilde{\theta}(k)\right), \\
& +\left(\left(I-\Omega^{T}(k) \Lambda_{\theta} \Omega(k)\right) \tilde{\theta}(k)\right)^{T}\left(\left(I-\Omega^{T}(k) \Lambda_{\theta} \Omega(k)\right) \tilde{\theta}(k)\right)-e_{f i}^{T}(k) S e_{f i}(k)-q \tilde{\theta}^{T} \tilde{\theta}, \\
= & -e_{f i}^{T}(k) Q_{0} e_{f i}(k)-\tilde{\theta}^{T}(k) \Omega^{T}(k)\left[q \Lambda_{\theta}^{T}(k) \Omega_{\theta} \Lambda_{\theta}(k)-S\right] \Omega(k) \theta(k) \\
& +2 e_{f i}^{T}(k) \Lambda^{T} S \Omega(k) \tilde{\theta}(k) .
\end{aligned}
$$

Considering (4.7) with $\varepsilon(k)=0$, from (4.11) we can deduce

$$
\begin{aligned}
\Delta V(k)= & \sum_{i=1}^{N} \Delta V_{i}(k), \\
= & \sum_{i=1}^{N}\left(-e_{f i}^{T}(k) Q_{0} e_{f i}(k)-\tilde{\eta}^{T}(k)\left[q \Lambda_{\theta}^{T}(k) \Omega_{\theta} \Lambda_{\theta}(k)-S\right] \tilde{\eta}(k)+2 e_{i}^{T}(k) \Lambda^{T} S \tilde{\eta}(k)\right), \\
\leq & \sum_{i=1}^{N}\left(-\lambda_{\min }\left(Q_{0}\right)\left\|e_{i}(k)\right\|^{2}-\left(q \beta^{2} \lambda_{\min }\left(Q_{\theta}\right)-\lambda_{\max }(S)\right)\|\tilde{\eta}(k)\|^{2}\right. \\
& \left.\quad+2 \lambda_{\max }(S)\|\Lambda\|\left\|e_{f i}(k)\right\|\|\tilde{\eta}(k)\|\right),
\end{aligned}
$$




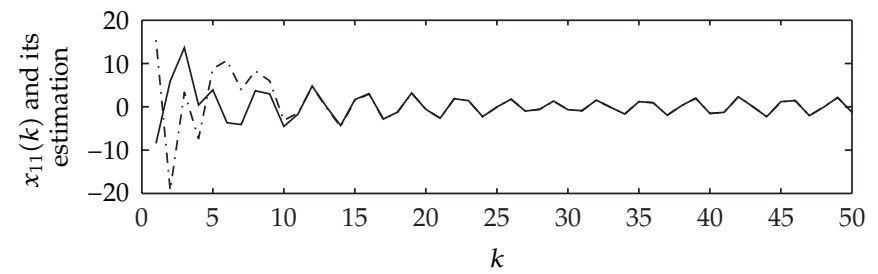

(a)

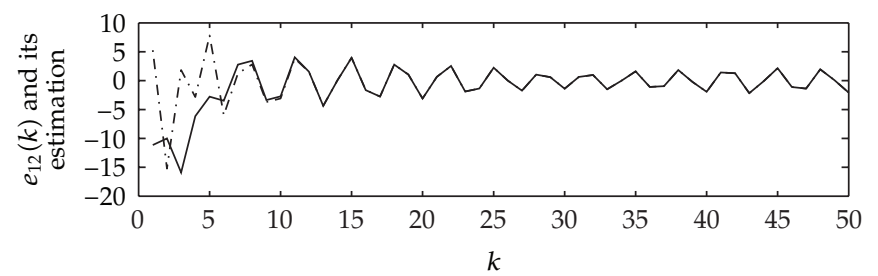

(b)

Figure 1: State curves of $x_{1}(k)$ and $\widehat{x}_{1}(k)$.

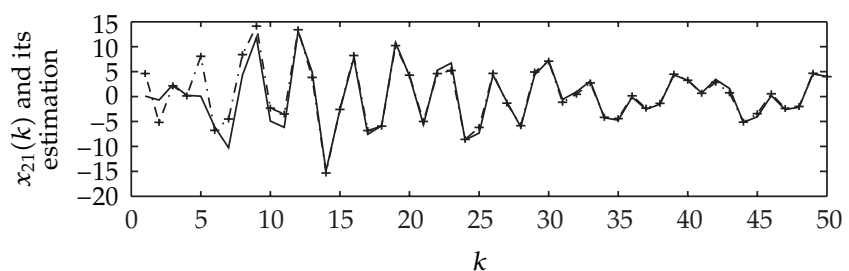

(a)

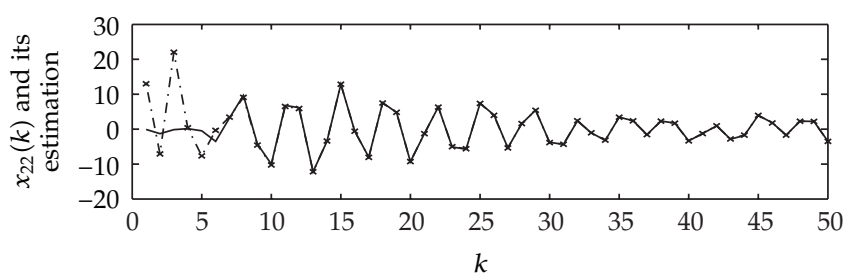

(b)

Figure 2: State curves of $x_{2}(k)$ and $\widehat{x}_{2}(k)$.

where $\left\|\Lambda_{\theta}\right\| \geq \beta>0 . \Delta V(k) \leq 0$ holds if $q$ satisfies the inequality

$$
q>\frac{\lambda_{\max }(S) \lambda_{\min }\left(Q_{0}\right)+\lambda_{\max }^{2}(S)\|\Lambda\|^{2}}{\lambda_{\min }\left(Q_{0}\right) \beta^{2} \lambda_{\min }\left(Q_{\theta}\right)}
$$

Since $V(k)$ is a decreasing and nonnegative function, it converges to a constant value as $k \rightarrow \infty$, hence $\Delta V(k) \rightarrow 0$. This implies that both $e_{f i}(k)$ and $\tilde{\eta}(k)$ remain bounded for all $k$, and $e_{i}(k)$ approaches to zero. 


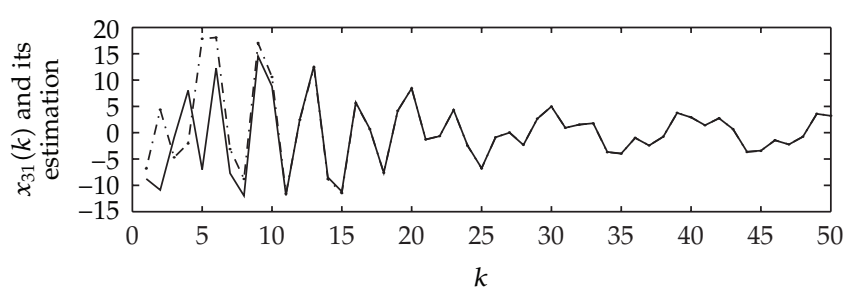

(a)

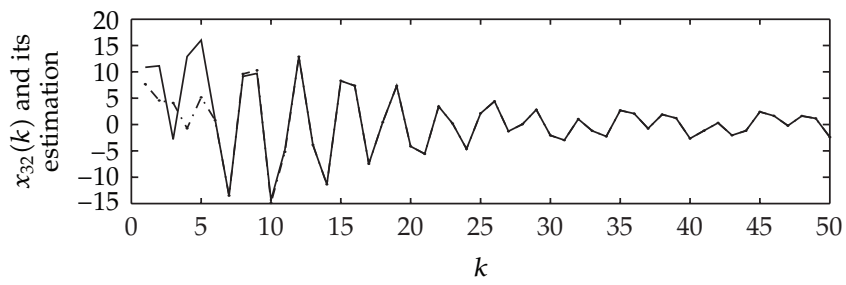

(b)

Figure 3: State curves of $x_{3}(k)$ and $\widehat{x}_{3}(k)$.

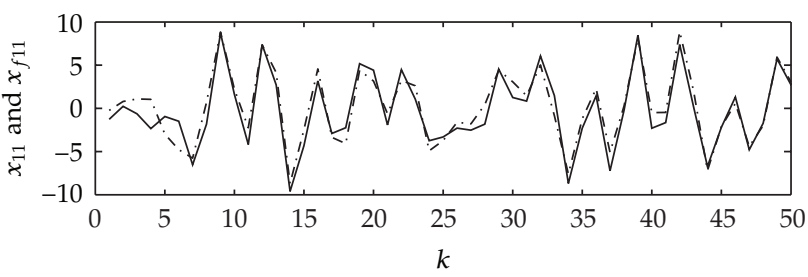

(a)

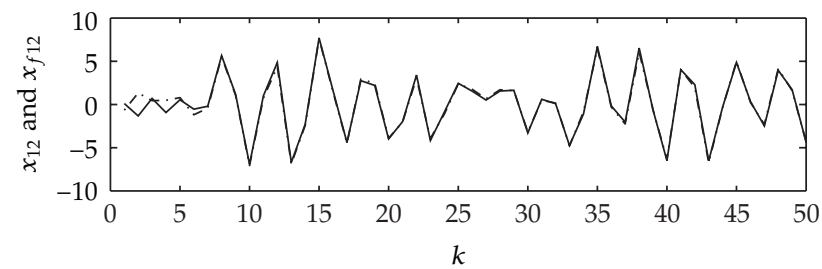

(b)

Figure 4: State curves of $x_{1}(k)$ and $\widehat{x}_{f 1}(k)$.

Once the residual vector $r_{i}(k)$ is computed at each step, a fault is declared if each component of $r_{i}(k)$ exceeds a suitably selected threshold. A priori selection of each threshold should be based on the expressions of the residuals vector. Namely, proper setting of the thresholds requires an accurate knowledge of the uncertainties influence on the residuals. However, this approach often leads to extremely conservative results. Therefore, an empirical approach may be pursued to set the residuals thresholds in alternative to (or in combination with) the approach based on a priori knowledge of the uncertainties. 


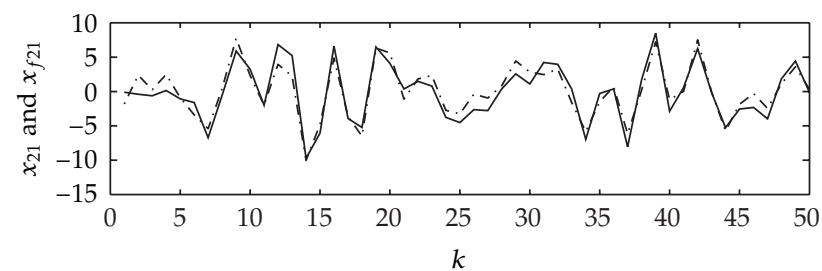

(a)

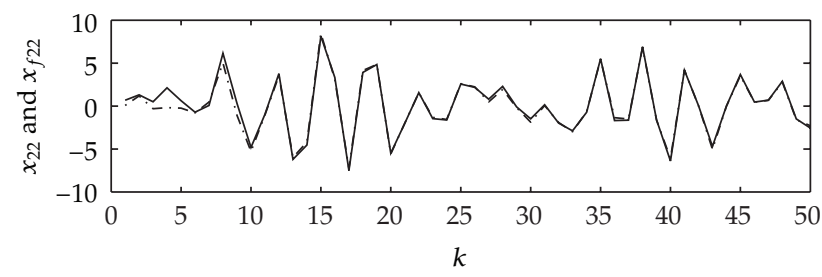

(b)

Figure 5: State curves of $x_{2}(k)$ and $\widehat{x}_{f 2}(k)$.

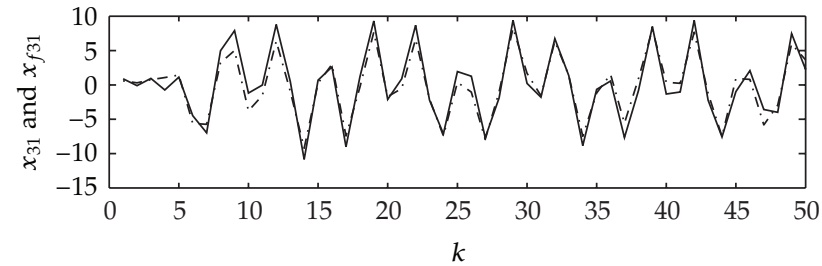

(a)

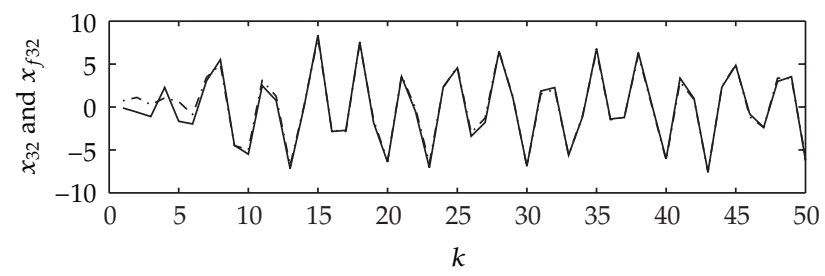

(b)

Figure 6: State curves of $x_{3}(k)$ and $\widehat{x}_{f 3}(k)$.

\section{Numerical Simulation}

In this section, we will use an example to show the effectiveness of the proposed result.

Example 5.1. Let us consider a complex system composed by systems (2.1) and (2.2) with $N=3$ and $n=2$. The system parameters in (2.1) and (2.2) are as follows, $A=\left[\begin{array}{cc}-0.8 & 0.9 \\ -0.85 & -0.1\end{array}\right]$, $\tau(k)=3+\left(1+(-1)^{k}\right) / 2, \Gamma=\operatorname{diag}(0.5,0.5), G_{i j}=0.1$ if $i \neq j$, and $G_{i j}=-0.2$ if $i=j . D_{i}=D=$ $\left[\begin{array}{cc}1 & 0 \\ 0.2 & 0\end{array}\right], B=\left[\begin{array}{cc}1 & 0.1 \\ 0.3 & 0\end{array}\right]$. The nonlinear vector-value function is given by $g\left(x_{i}(k)\right)=\left(-0.05 x_{i 1}(k)+\right.$ $\left.\tanh \left(0.02 x_{i 1}(k)\right)+0.02 x_{i 2}(k), 0.095 x_{i 2}(k)-\tanh \left(0.075 x_{i 2}(k)\right)\right)^{T}, i=1,2,3$. Obviously, $\Delta_{1}=$ $\left[\begin{array}{cc}-0.05 & 0.02 \\ 0 & 0.095\end{array}\right], \Delta_{2}=\left[\begin{array}{cc}-0.03 & 0.02 \\ 0 & 0.02\end{array}\right]$. 


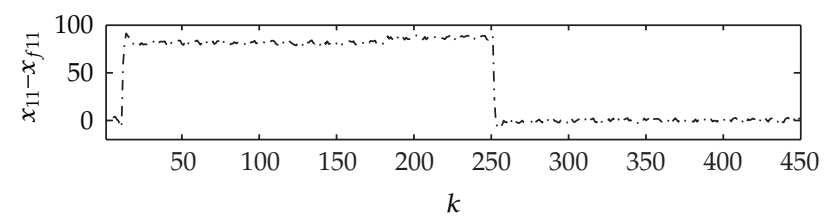

(a)

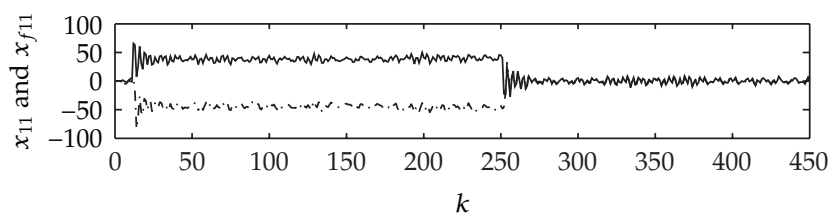

(b)

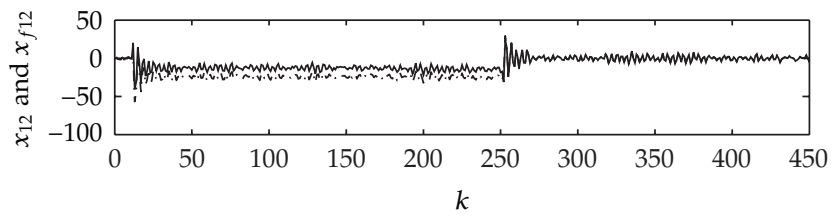

(c)

Figure 7: Error curves of $x_{11}(k)-\widehat{x}_{f 11}(k)$ in the faulty case.

By using the Matlab LMI Toolbox, using Theorem 3.2 to solve inequalities (3.15), the feasible solutions are as follows: $P=\left[\begin{array}{lll}0.1225 & 0.0189 \\ 0.0189 & 0.4993\end{array}\right], Q=\left[\begin{array}{lll}0.9413 & 0.4806 \\ 0.4806 & 1.9926\end{array}\right], Y=\left[\begin{array}{lll}0.1120 & 0.0224 \\ 0.3722 & 0.0744\end{array}\right]$, and

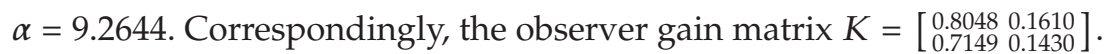

When $u(k)=\left[\sin \left(6 \pi k t_{s}\right)+4 \cos \left(2 \pi k t_{s}\right) ; 3 \cos \left(7 \pi k t_{s}\right)\right], t_{s}=0.1$, and the initial states are randomly chosen, using the state observer (2.10), the state curves are depicted in Figures 1-3, respectively. Obviously, the estimated states can track the system states accurately.

When $\eta(k)=\left[2 \cos \left(2 \pi x_{i 1}\right) ; 0.2 \cos \left(2 \pi x_{i 2}\right)\right], f\left(k, x_{i}(k)\right)=0$, and the gain matrix $K_{o}$ in (4.1) is chosen as $K_{o}=\left[\begin{array}{ll}-0.4687 & 0.6941 \\ -0.7354 & -0.3082\end{array}\right]$ (in which the eigenvalues of $\Lambda=A-K_{o}$ are $0.2884+$ $0.1310 i$ and $0.2884-0.1310 i$, resp.), the states of the diagnosis observe (4.1) and the system states (2.1) are depicted in Figures 4-6, respectively.

When the following faults occur in the first node system:

$$
\begin{gathered}
\delta u_{11}=60\left(1-\frac{e^{-\left(k t_{s}-1\right)}}{0.002}\right), \quad k t_{s} \geq 1, \\
\delta u_{12}=40\left(1-e^{-\left(k t_{s}-3\right) / 0.08}\right), \quad k t_{s} \geq 15, \\
\delta u_{11}=\delta u_{12}=0, \quad k t_{s} \geq 25,
\end{gathered}
$$

the state errors of $x_{1}(k)-\widehat{x}_{f 1}(k)$, diagnosis observer states $\widehat{x}_{f 1}$, and the system state $x_{1}(k)$ are depicted in Figures 6-8, respectively. The residual curves are depicted in Figure 9. If we choose suitably the fault threshold, we can accurately detect the fault.

Note that we assume that the fault occurs in the first node system. Since three node systems constitute a complex interconnected network, the fault in the first node system will affect the other two systems. The state response curves of the other systems $x_{2}(k)$ and $x_{3}(k)$ 


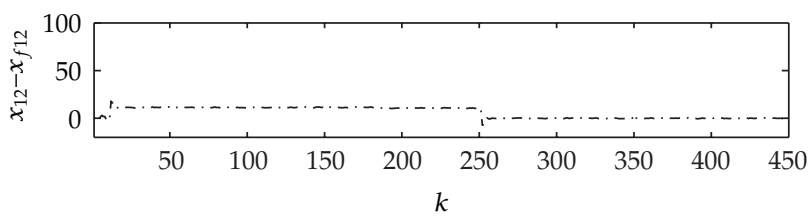

(a)

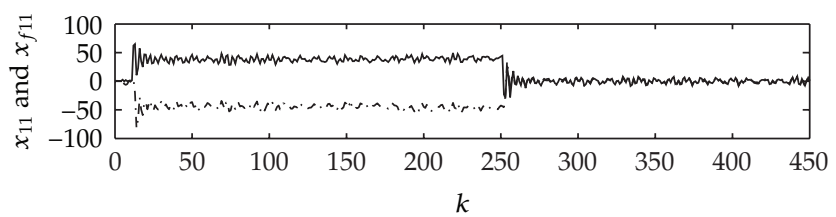

(b)

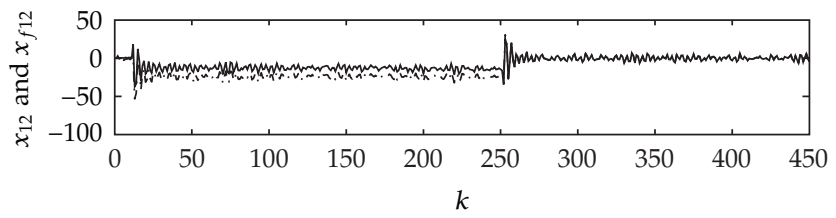

(c)

Figure 8: Error curves of $x_{12}(k)-\widehat{x}_{f 12}(k)$ in the faulty case.

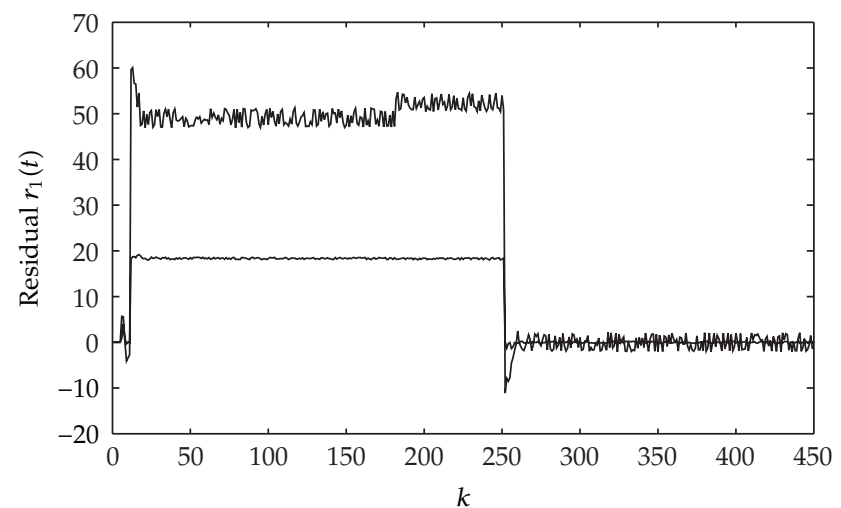

Figure 9: Residual curves of first node system in the faulty case.

are depicted in Figures 10-11, respectively. Obviously, the states in the other two systems will!be influenced by the first faulty system $x_{1}(k)$. In this case, if we judge whether the system is healthy or not, it is not sufficient to detect the fault by the other system. However, if we observe the residual curves of the other two systems, see Figure 12, we may judge the fault location by choosing a suitable logic rule, which is out of the coverage in the paper.

\section{Conclusions}

In this paper, a state observer is constructed for a class of discrete-time delayed complex interconnected networks with linear coupling based on LMI method. Then on the availability 


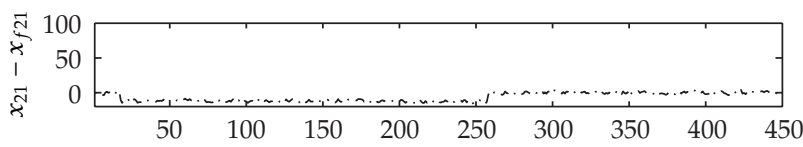

(a)

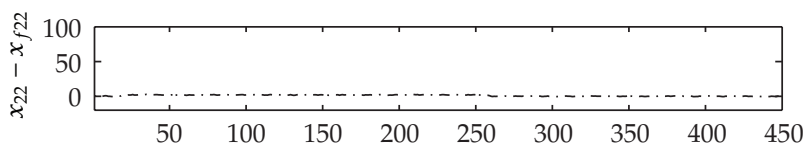

(b)

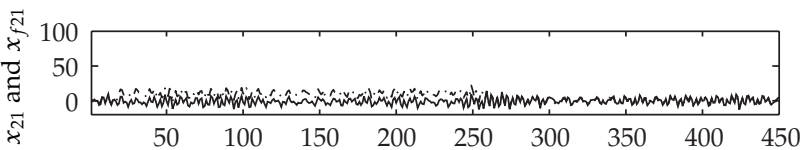

(c)

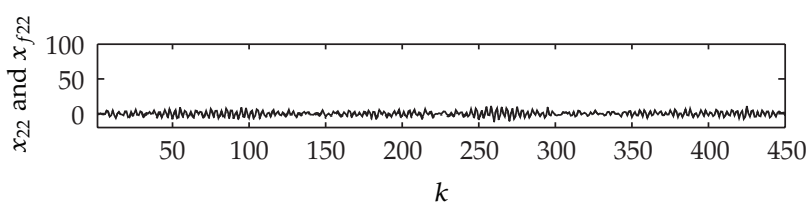

(d)

Figure 10: Error curves of $x_{2}(k)-\widehat{x}_{f 2}(k)$ in the faulty case.

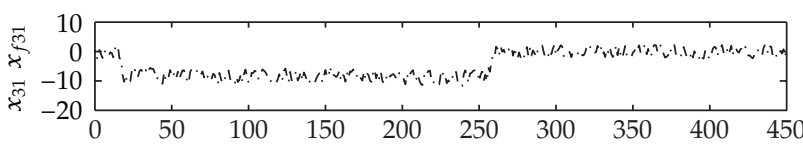

(a)

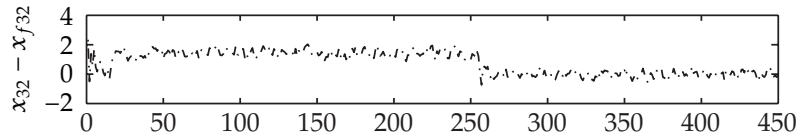

(b)

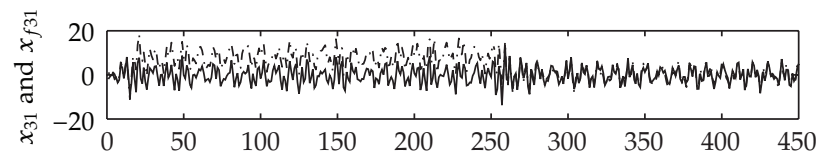

(c)

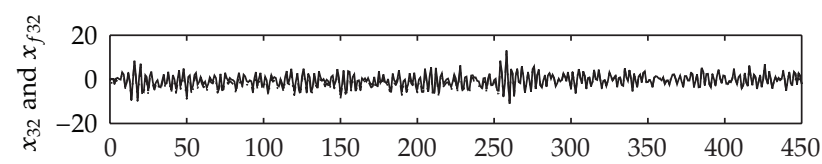

(d)

Figure 11: Error curves of $x_{3}(k)-\widehat{x}_{f 3}(k)$ in the faulty case. 


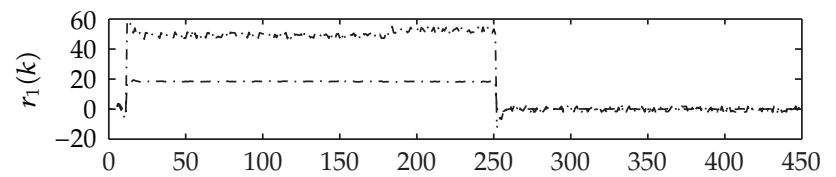

(a)

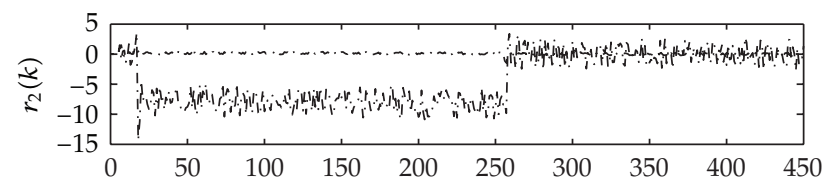

(b)

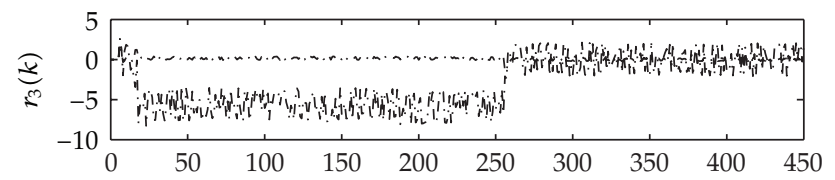

(c)

Figure 12: Residual curves of the three systems in the faulty case.

of the system states, an adaptive fault diagnosis observer is designed to realize the actuator fault detection. The uncertainty of the system is modelled by an online approximation technique, and the stability of the residual system and approximation system are analyzed. Finally, a numerical example is used to validate the proposed result. Note that the proposed state observer and the fault diagnosis observer can only implement the diagnosis task. How to realize the fault identification will be further studied.

\section{Acknowledgments}

This work was supported by the National Natural Science Foundation of China under Grants 61074073 and 61034005, Program for New Century Excellent Talents in University of China (NCET-10-0306), and the Fundamental Research Funds for the Central Universities under Grants N110504001 and N100104102.

\section{References}

[1] J. Chen and R. Patton, Robust Model-Based Fault Diagnosis for Dynamic Systems, Kluwer, Boston, Mass, USA, 1999.

[2] S. Narasimhan and G. Biswas, "Model-based diagnosis of hybrid systems," IEEE Transactions on Systems, Man, and Cybernetics A, vol. 37, no. 3, pp. 348-361, 2007.

[3] J. Stoustrup and H. H. Niemann, "Fault estimation-a standard problem approach," International Journal of Robust and Nonlinear Control, vol. 12, no. 8, pp. 649-673, 2002.

[4] F. Basile, P. Chiacchio, and G. De Tommasi, "An efficient approach for online diagnosis of discrete event systems," IEEE Transactions on Automatic Control, vol. 54, no. 4, pp. 748-759, 2009.

[5] N. Meskin and K. Khorasani, "Robust fault detection and isolation of time-delay systems using a geometric approach," Automatica, vol. 45, no. 6, pp. 1567-1573, 2009.

[6] Q. Yang and Y. Sun, "Automated fault accommodation for discrete-time systems using online approximators," in Proceedings of the 30th Chinese Control Conference, Yantai, China, July 2011. 
[7] R. M. G. Ferrari, T. Parisini, and M. M. Polycarpou, "Distributed fault diagnosis of large-scale discretetime nonlinear systems: new results on the isolation problem," in Proceedings of the 49th IEEE Conference on Decision and Control (CDC '10), pp. 1619-1626, Hilton Atlanta Hotel, Atlanta, Ga, USA, December 2010.

[8] H. Yang, B. Jiang, and V. Cocquempot, "Supervisory fault-tolerant regulation for nonlinear systems," Nonlinear Analysis, vol. 12, no. 2, pp. 789-798, 2011.

[9] I. Samy, I. Postlethwaite, and D.-W. Gu, "Survey and application of sensor fault detection and isolation schemes," Control Engineering Practice, vol. 19, no. 7, pp. 658-674, 2011.

[10] R. Patton, P. Frank, and R. Clark, Fault Diagnosis in Dynamic Systems: Theory and Application, Prentice Hall, 1989.

[11] M. Blanke, M. Kinnaert, J. Lunze, and M. Staroswiecki, Diagnosis and Fault Tolerant Control, Berlin, Germany, 2006.

[12] R. Isermann, "Process fault detection based on modeling and estimation methods—a survey," Automatica, vol. 20, no. 4, pp. 387-404, 1984.

[13] J. J. Gertler, "Survey of model-based failure detection and isolation in complex plants," IEEE Control Systems Magazine, vol. 8, no. 6, pp. 3-11, 1988.

[14] P. M. Frank, “Fault diagnosis in dynamic systems using analytical and knowledge-based redundancy. A survey and some new results," Automatica, vol. 26, no. 3, pp. 459-474, 1990.

[15] V. Venkatasubramanian, R. Rengaswamy, K. Yin, and S. N. Kavuri, "A review of process fault detection and diagnosis part I: quantitative model-based methods," Computers and Chemical Engineering, vol. 27, no. 3, pp. 293-311, 2003.

[16] B. Jiang, M. Staroswiecki, and V. Cocquempot, "Fault accommodation for nonlinear dynamic systems," IEEE Transactions on Automatic Control, vol. 51, no. 9, pp. 1578-1583, 2006.

[17] B. Jiang and F. N. Chowdhury, "Fault estimation and accommodation for linear MIMO discrete-time systems," IEEE Transactions on Control Systems Technology, vol. 13, no. 3, pp. 493-499, 2005.

[18] K. Zhang, B. Jiang, and P. Shi, "Fast fault estimation and accommodation for dynamical systems," IET Control Theory and Applications, vol. 3, no. 2, pp. 189-199, 2009.

[19] C. Langbort, R. S. Chandra, and R. D'Andrea, "Distributed control design for systems interconnected over an arbitrary graph," IEEE Transactions on Automatic Control, vol. 49, no. 9, pp. 1502-1519, 2004.

[20] R. M. G. Ferrari, T. Parisini, and M. M. Polycarpou, “Distributed fault diagnosis with overlapping decompositions: an adaptive approximation approach," IEEE Transactions on Automatic Control, vol. 54, no. 4, pp. 794-799, 2009.

[21] H. Zhang, D. Yang, and T. Chai, "Guaranteed cost networked control for T-S fuzzy systems with time delays," IEEE Transactions on Systems, Man and Cybernetics C, vol. 37, no. 2, pp. 160-172, 2007.

[22] Z. Wang, Y. Wang, and Y. Liu, "Global synchronization for discrete-time stochastic complex networks with randomly occurred nonlinearities and mixed time delays," IEEE Transactions on Neural Networks, vol. 21, no. 1, pp. 11-25, 2010.

[23] Z. Li and G. Chen, "Global synchronization and asymptotic stability of complex dynamical networks," IEEE Transactions on Circuits and Systems II, vol. 53, no. 1, pp. 28-33, 2006.

[24] D. Ding, Z. Wang, B. Shen, and H. Shu, " $H_{\infty}$ state estimation for discrete-time complex networks with randomly occurring sensor saturations and randomly varying sensor delays," IEEE Transactions Neural Networks and Learning Systems, vol. 23, no. 5, pp. 725-726, 2012.

[25] X. F. Wang and G. Chen, “Complex networks: small-world, scale-free and beyond," IEEE Circuits and Systems Magazine, vol. 3, no. 1, pp. 6-20, 2003.

[26] Y. Liu, Z. Wang, J. Liang, and X. Liu, "Synchronization and state estimation for discrete-time complex networks with distributed delays," IEEE Transactions on Systems, Man, and Cybernetics B, vol. 38, no. 5, pp. 1314-1325, 2008.

[27] Z. Wang, H. Zhang, and B. Jiang, "LMI-based approach for global asymptotic stability analysis of recurrent neural networks with various delays and structures," IEEE Transactions on Neural Networks, vol. 22, no. 7, pp. 1032-1045, 2011.

[28] D. Gong, H. Zhang, Z. Wang, and D. Ma, "Synchronization criteria for an array of neutral-type neural networks with hybrid coupling: a novel analysis approach," Neural Processing Letters, vol. 35, no. 1, pp. 29-45, 2012.

[29] D. Gong, H. Zhang, Z. Wang, and B. Huang, "Novel synchronization analysis for complex networks with hybrid coupling by handling multitude Kronecker product terms," Neurocomputing, vol. 82, pp. 14-20, 2012. 
[30] X. Jin and G. Yang, "Adaptive synchronization of a class of uncertain complex networks against network deterioration," IEEE Transactions on Circuits and Systems I, vol. 58, no. 6, pp. 1396-1409, 2011.

[31] X. Jin, G. Yang, and W. Che, "Adaptive pinning control of deteriorated nonlinear coupling networks with circuit realization," IEEE Transactions on Neural Networks and Learning Systems, vol. 23, no. 9, pp. 1345-1355, 2012.

[32] Y.-W. Wang, J.-W. Xiao, and H. O. Wang, "Global synchronization of complex dynamical networks with network failures," International Journal of Robust and Nonlinear Control, vol. 20, no. 15, pp. 16671677, 2010.

[33] K. Zhang and L. Li, "Robust adaptive decentralized control for a class of networked large-scale systems with sensor network failures," in Proceeding of the Chinese Control and Decision Conference (CCDC '11), pp. 2366-2370, Mianyang, China, 2011.

[34] H. Zhang and Y. Quan, "Modeling, identification, and control of a class of nonlinear systems," IEEE Transactions on Fuzzy Systems, vol. 9, no. 2, pp. 349-354, 2001.

[35] M. M. Polycarpou and A. J. Helmicki, "Automated fault detection and accommodation: a learning systems approach," IEEE Transactions on Systems, Man and Cybernetics, vol. 25, no. 11, pp. 1447-1458, 1995.

[36] A. T. Vemuri and M. M. Polycarpou, "Robust nonlinear fault diagnosis in input-output systems," International Journal of Control, vol. 68, no. 2, pp. 343-360, 1997.

[37] A. B. Trunov and M. M. Polycarpou, "Automated fault diagnosis in nonlinear multivariable systems using a learning methodology," IEEE Transactions on Neural Networks, vol. 11, no. 1, pp. 91-101, 2000.

[38] A. T. Vemuri, "Sensor bias fault diagnosis in a class of nonlinear systems," IEEE Transactions on Automatic Control, vol. 46, no. 6, pp. 949-954, 2001. 


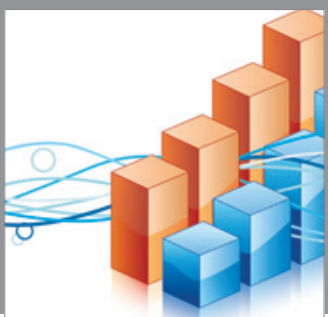

Advances in

Operations Research

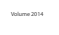

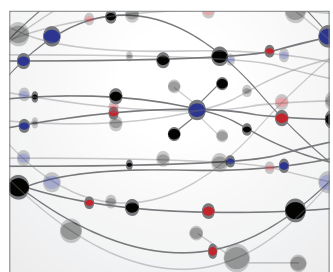

\section{The Scientific} World Journal
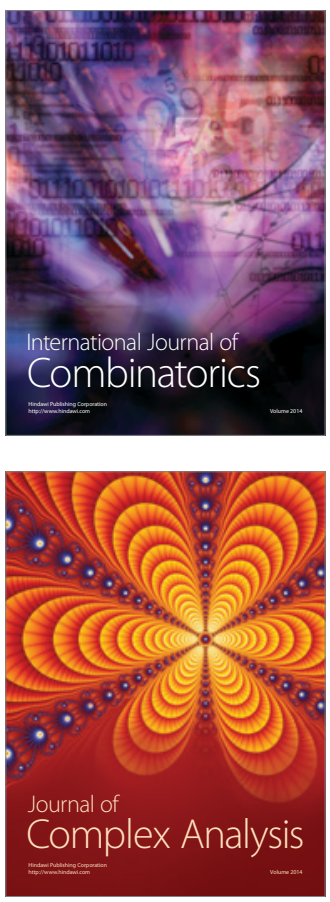

International Journal of

Mathematics and

Mathematical

Sciences
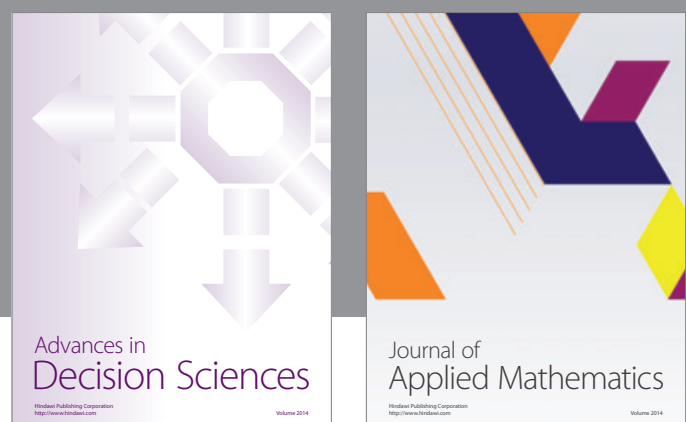

Journal of

Applied Mathematics
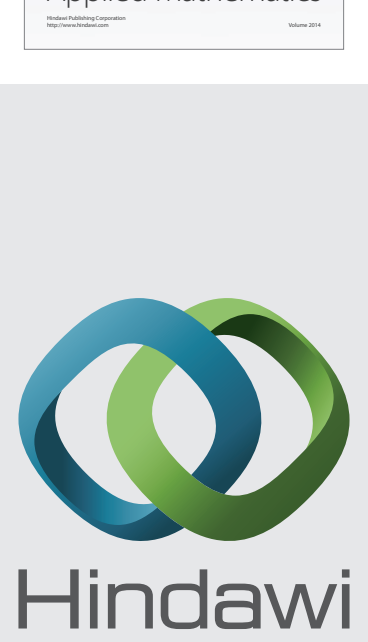

Submit your manuscripts at http://www.hindawi.com
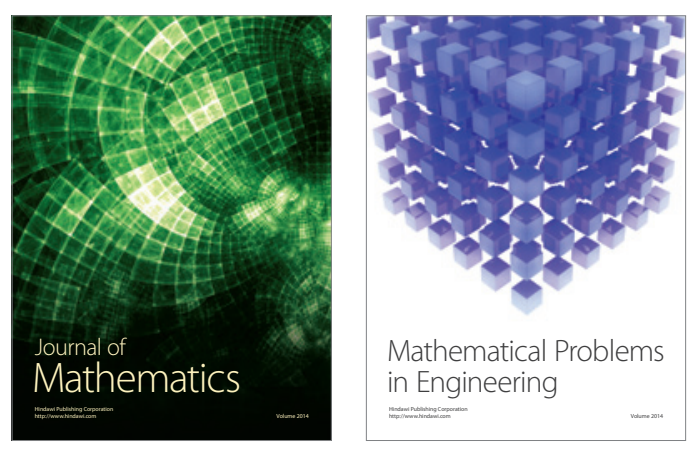

Mathematical Problems in Engineering
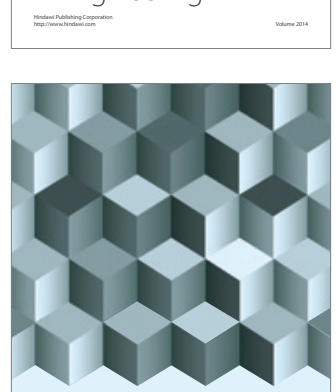

Journal of

Function Spaces
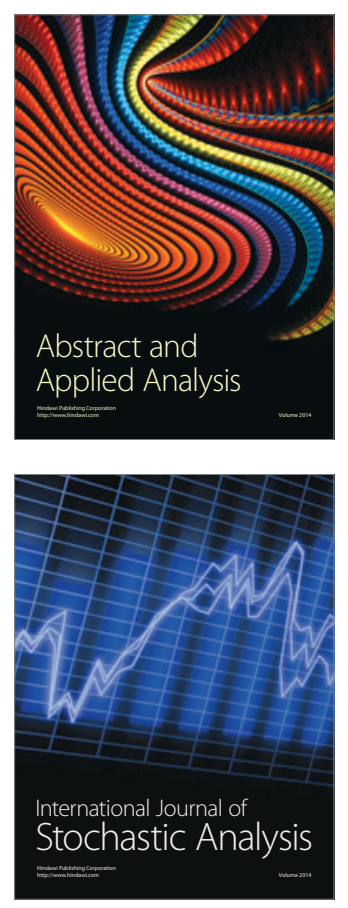

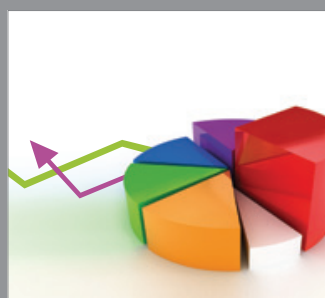

ournal of

Probability and Statistics

Promensencen
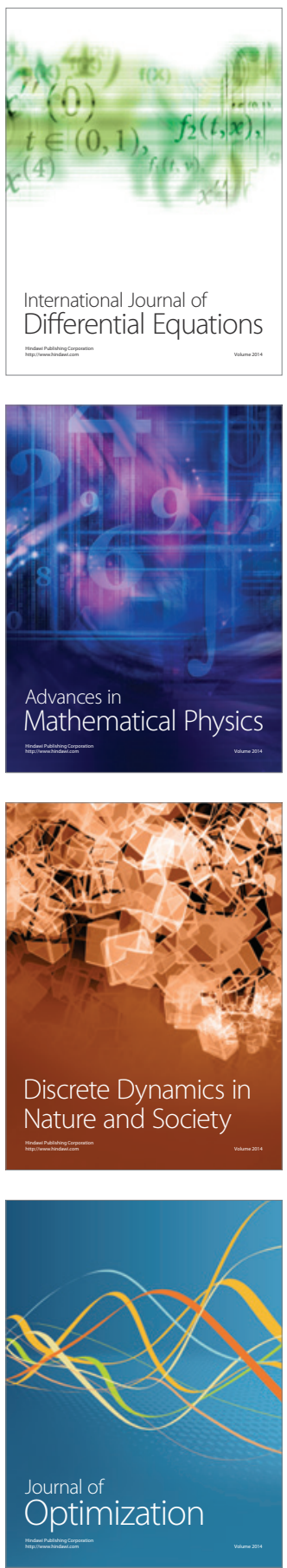